\title{
【Transaction】
}

\section{Development of Kinematic Soft Dummy and Application on Clothing Pressure Measurement of Men's Suit Pants}

\author{
Hiroyuki Kanai*1,2,\#, Kentaro Ogawa*1, Tetsu Sasagawa*3, \\ Kiyohiro Shibata*3, and Kei Kawauchi*4 \\ ${ }^{* 1}$ Faculty of Textile Science and Technology, Shinshu university, 3-15-1 Tokida, Ueda, Nagano 386-8567, Japan \\ ${ }^{* 2}$ Institute for Fiber Engineering, Shinshu University, 3-15-1 Tokida, Ueda, Nagano 386-8567, Japan \\ *3 Aoki holdings Ltd., 60-1 Aza-shimoyanaginouchi, Meichi, Ichinomiya, Aichi 494-0012, Japan \\ ${ }^{* 4}$ Kato Tech co., Ltd., 26 Karato-cho, Nishikujo, Minami-ku, Kyoto 601-8447 Japan
}

\begin{abstract}
The quantitative evaluation of clothing comfort is essential in the clothing industry. Garment constraint is caused by its tension along the curvature of the human body when in motion. Hence, the measurement of clothing pressure using test persons has been considered a representative tool for evaluating the motion adaptability of garments. Nevertheless, errors are caused by the individual differences among humans as well as the motion variance. In this study, a kinematic soft dummy model was designed and developed to measure clothing pressure. First, a model was selected that approximated the standard body proportions and dimensions of Japanese males in their 20s; the external mold was produced using gypsum. Second, a mechanical skeleton was inserted into the external mold, and the dummy was formed around the skeleton using a specific stiffness polyurethane simulating the modulus of human tissues. Third, the kinematic function was implemented through a control system that enabled the soft dummy to conduct "one leg raising". Finally, the clothing pressure of the kinematic soft dummy was measured; it was fitted with men's suit pants that consisted of six worsted fabrics with different elongation ratios. Our results confirmed a particularly high correlation coefficient for the clothing pressure of the kinematic soft dummy and the test persons. They also confirmed a particularly high correlation coefficient for the clothing pressure of the kinematic soft dummy and the subjective evaluation by test persons. Therefore, the clothing pressure measurement with a kinematic soft dummy could be a novel and effective method for comfort evaluation.
\end{abstract}

(Received 5 August, 2021; Accepted 9 September, 2021)

\section{Introduction}

The development of a right tool and metric for evaluating clothing comfort has received increasing interest. In this study, a dummy of the lower half of the human body was developed. The dummy was produced using a soft material and integrated with a kinematic function (hereinafter, referred to as "kinematic soft dummy"). The kinematic soft dummy was expected to reproduce the distribution of clothing pressure observed on test persons after placing a garment on it.

A tool of this type could be applied to evaluate the motion adaptability of garments. In particular, it could be useful to fashion designers, textile makers, and pattern makers for always integrating their perception of motion adaptability into the design, development, and production stages, respectively. It could also enable customers to know the assessment results of the motion adaptability of a garment when they shop online, which is very useful for reducing the high return rate caused by unmet expectations.

For the design and development of a dummy suitable for measuring clothing pressure, four important functions should be implemented: (1) Accurate replication of the body proportions and dimensions of a test person model; this not only offers high accuracy to the result of clothing pressure but also universalizes the result to be comparable. Such a dummy is called an anthropomorphic dummy.

(2) Replication of body stiffness, which highly impacts the action and reaction of stress or its

\# corresponding author: Hiroyuki Kanai (E-mail: kanai@shinshu-u.ac.jp) 
relaxation. As a result, it offers an accurate reproduction of the garment deformation.

(3) Realization of posture variation, such as upright, sitting, or supine, by inserting a mechanical structure equivalent to the skeleton of a human body in the dummy. The posture variation enables the measurement of the clothing pressure under real-life conditions. For example, the clothing pressure in the supine position should be measured to evaluate a sleeping garment.

(4) Realization of movability. Clothing pressure is caused by the tension along the curvature of the human body when in motion. The implementation of the dummy's movability offers the reproducibility of the garment deformation, and thus, stability in the clothing pressure measurement.

Several studies have been conducted to design a dummy with these requirements [1-15]. An anthropomorphic dummy has been widely used as an alternative to the human body to measure clothing pressure. Most research studies used an anthropomorphic dummy with a hard surface, which approximately matches the industrial sizing. Jing et al. used a commercial hard body dummy to measure clothing pressure while wearing pressure stockings [1]. However, some commercial anthropomorphic dummies are often not applicable as standard body proportions. Liu et al. compared the popular mannequin with the China National Standard of garment sizing system GB/T 1335.1 and reported that the mannequin's back is too thick and too straight [2]. If the clothing pressure is measured using the anthropomorphic dummy, it is impacted by the incorrect body proportions and dimensions.

To address these issues, a specialized dummy that precisely corresponds to the shape and size of the Japanese standardized body provided by the Research Institute of Human Engineering for Quality Life (HQL) has been developed [3]. Sugaya et al. measured the clothing pressure acting along the shoulder belt by putting a backpack onto a specialized dummy [4].

In contrast, industrialists and researchers proposed a dummy with soft skin. The first patent relevant to soft mannequin was published in 1975 [5] and developed competitively [6-9]. Yu et al. proposed a female-shaped soft dummy that consisted of an elaborate skeleton and three different materials to anatomically simulate the modulus distribution. For example, the modulus of the abdomen was softer than that of the main body. The modulus of the surface skin was also softer than that of the main body. Then, they compared the clothing pressure of the dummy with that of the human body and reported good agreement [10-11]. Tamura et al. also proposed a female-shaped soft dummy. They simultaneously produced a soft dummy and a hard dummy with the same mold. Subsequently, they compared the clothing pressure values obtained from the soft dummy, hard dummy, and model. Their results proved good agreement between the clothing pressure on the soft dummy and that on the models [12]. Furthermore, they implemented the posture variation of the dummy and measured the clothing pressure in upright and sitting postures when the dummy was wearing a diaper for adults [13]. Wang et al. proposed a shape-changing dummy with a soft surface. They reported that it was an applicable tool for assessing the fitting feature of compression clothing in various body shapes [14-15].

The soft dummies proposed in previous research [10-13] consisted of an elaborate skeleton model and multiple soft materials differing in their modulus. According to their proposed method, a dummy that implements the modulus distribution due to the anatomical structure can be created. However, a skilled technique and extensive experience are required to create a dummy with a complex structure. We deemed that this specification was extremely demanding in comparison with the delivered effect. In addition, according to Tamura et al., the soft dummy that involves the elaborate skeleton made by glass fiber reinforced plastics is fragile; in particular, dislocation of the hip joint often occurred in their research [12]. They also reported that the fragility of the elaborate skeleton and the heaviness of the dummy resulted in difficulties in posture variability and movability [12]. Owing to these technical issues, the application of the proposed soft dummy was limited to the static measurement of clothing pressure due to the circumferential stress of compression clothing.

However, industrialists and researchers should implement a kinematic function into the soft dummy and measure clothing pressure to evaluate the motion adaptability of garments.

In this study, a kinematic soft dummy was developed by replicating the shape, dimensions, and stiffness of the human body. Moreover, this study aimed for posture variability and movability. In 
addition, men's suit pants that consisted of six graded worsted fabrics with different elongation ratios (EMTs) were used as experimental samples, and measured the clothing pressure on a dummy to validate the effect as an evaluation tool for the motion adaptability of garments.

This study makes a novel contribution to the literature by proposing a prediction method of garment motion adaptability from the clothing pressure measurement with a kinematic soft dummy.

The study contributes significantly to the literature because the kinematic soft dummy can be a useful tool for fashion designers, textile makers, and pattern makers to integrate their perceptions of motion adaptability into the design, development, and production stages, respectively.

\section{Method}

\subsection{Design and development of kinematic soft dummy}

\subsubsection{Selection of model and its replication}

A representative model was selected from nine Japanese males in their 20s. The anthropometric dimensions of 25 parts in the lower half of the body, as shown in Fig. 1 (a) and Table 1, were obtained through the contact method using a Martin-type anthropometer [17]. Subsequently, an anthropometric dimension was assessed to match the average of Japanese males in their 20s. Particularly, the standard band (hereinafter, referred to as " $S B$ ") was set on each part using the databases on anthropometry obtained from two Japanese research institutes (i.e., HQL and Advanced Industrial Science and Technology (AIST)). The mean value (i.e., $\bar{x}_{A I S T}$ and $\bar{x}_{H Q L}$ ) and standard deviation (i.e., $\pm S T D_{A I S T}$ and $\pm S T D_{H O L}$ ) were calculated

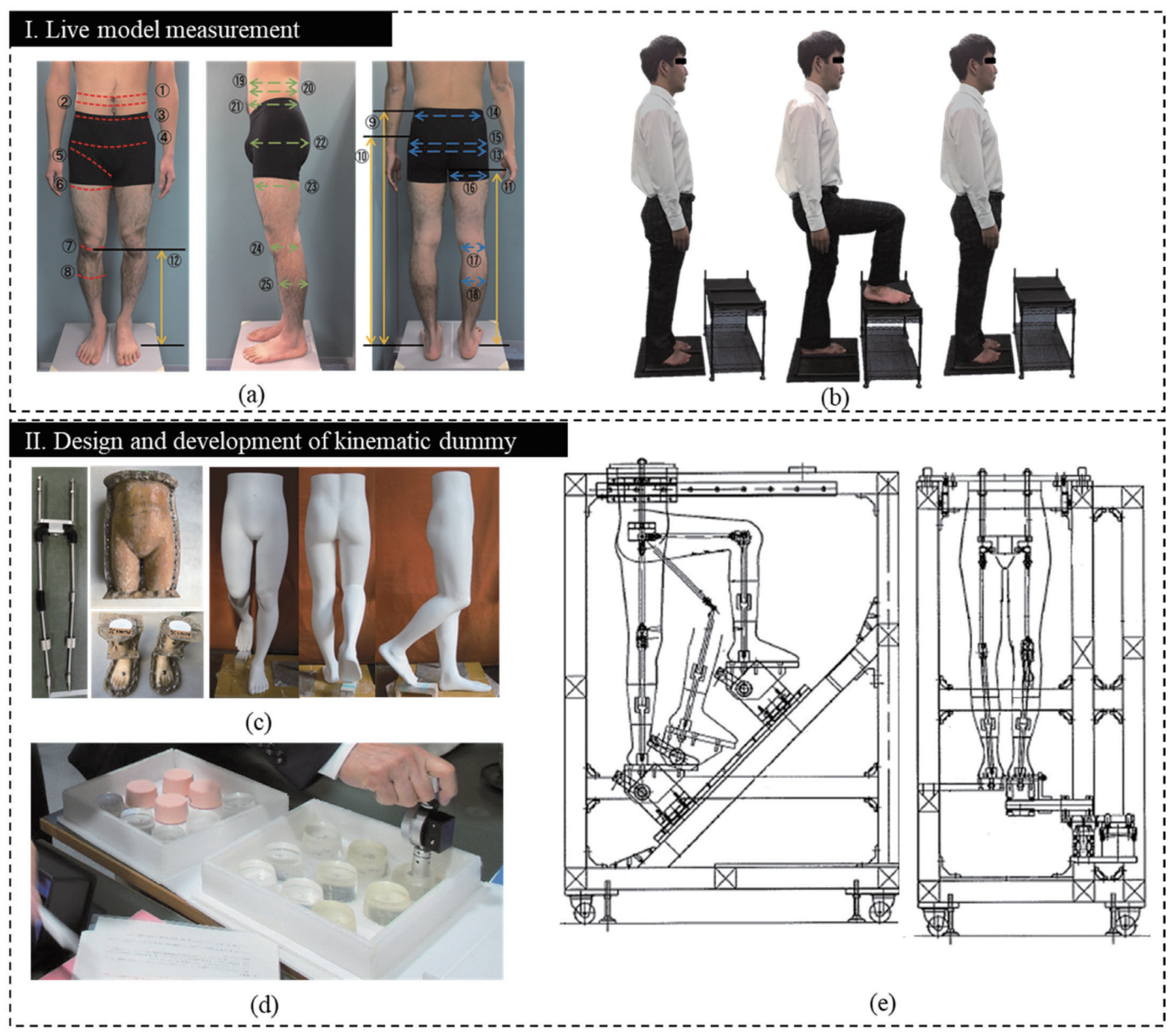

Fig. 1 Design of the kinematic soft dummy: (a) Anthropometry of a model. (b) Motion test. (c) An external mold obtained from model, and a mechanical structure. (d) Rigidity test of some PU specimens with varying proportions of polyol and additives. (e) Integration of the kinematic function. 
Table 1 Anthropometry of test persons and selection of a model for the replication of kinematic soft dummy

\begin{tabular}{|c|c|c|c|c|c|c|c|c|c|c|c|}
\hline \multicolumn{2}{|r|}{ Part } & \multirow{2}{*}{$\begin{array}{c}S B \\
67.7-81.5\end{array}$} & \multirow{2}{*}{$\begin{array}{l}\text { Panel1 } \\
65.2\end{array}$} & \multirow{2}{*}{$\frac{\text { Panel2 }}{6.5}$} & \multirow{2}{*}{$\begin{array}{c}\text { Panel3 } \\
\underline{76.2} \\
\end{array}$} & \multirow{2}{*}{$\begin{array}{c}\text { Panel4 } \\
\underline{79.5} \\
\end{array}$} & \multirow{2}{*}{$\begin{array}{c}\text { Panel5 } \\
67.5 \\
\end{array}$} & \multirow{2}{*}{$\begin{array}{c}\text { Panel6 } \\
66.5\end{array}$} & \multirow{2}{*}{$\begin{array}{r}\text { Panel7 } \\
\underline{\mathbf{7 2 . 0}} \\
\end{array}$} & \multirow{2}{*}{$\begin{array}{c}\text { Panel8 } \\
\underline{\mathbf{7 5 . 6}} \\
\end{array}$} & \multirow{2}{*}{$\begin{array}{c}\text { Panel9 } \\
\underline{\mathbf{8 0 . 5}}\end{array}$} \\
\hline 1 & Waist circumference & & & & & & & & & & \\
\hline 2 & Abdominal extension circumference & $74.9-86.0$ & 66.1 & 67.0 & $\underline{76.8}$ & $\underline{83.5}$ & 70.4 & 68.3 & $\underline{75.1}$ & $\underline{81.7}$ & $\underline{81.3}$ \\
\hline 3 & Iliocristal circumference & $74.5-87.0$ & $\mathbf{7 7 . 5}$ & 71.7 & $\underline{82.3}$ & $\underline{86.1}$ & 71.5 & $\underline{76.0}$ & $\underline{81.0}$ & $\underline{84.2}$ & $\underline{83.5}$ \\
\hline 4 & Buttock circumference & $86.6-95.9$ & $\underline{87.0}$ & 84.2 & $\underline{91.5}$ & 98.8 & $\underline{89.5}$ & 84.2 & $\underline{90.5}$ & $\underline{93.6}$ & 91.2 \\
\hline 5 & Inguinal circumference & $59.3-67.9$ & 54.6 & 54.0 & $\underline{61.8}$ & $\underline{67.7}$ & 57.0 & 57.2 & $\underline{61.7}$ & $\underline{65.3}$ & $\underline{63.3}$ \\
\hline 6 & Thigh circumference & $50.2-58.5$ & 48.9 & 46.5 & $\underline{\mathbf{5 3 . 3}}$ & 59.2 & $\underline{50.8}$ & 47.7 & $\underline{52.9}$ & $\underline{57.5}$ & $\underline{55.3}$ \\
\hline 7 & Knee circumference & $34.2-37.7$ & $\underline{34.8}$ & $\underline{35.0}$ & 38.3 & 40.1 & $\underline{34.4}$ & $\underline{34.8}$ & $\underline{36.4}$ & 38.6 & $\underline{34.5}$ \\
\hline 8 & Calf circumference & $34.3-39.1$ & 31.6 & 33.1 & $\underline{\underline{36.2}}$ & 39.2 & 34.2 & 34.0 & $\underline{36.5}$ & $\underline{38.3}$ & $\underline{35.8}$ \\
\hline 9 & Tliocristal height & $96.3-101.1$ & 88.6 & $\underline{100.7}$ & $\underline{97.2}$ & $\underline{100.3}$ & $\underline{96.9}$ & 88.2 & $\underline{98.4}$ & $\underline{97.2}$ & 91.3 \\
\hline 10 & Trochanterion height & $83.4-87.7$ & 78.5 & 93.3 & 88.9 & $\underline{86.0}$ & 83.2 & 81.8 & 91.8 & 90.8 & $\underline{85.3}$ \\
\hline 11 & Crotch height & $74.6-79.9$ & 72.8 & 85.2 & 80.0 & 78.4 & $\underline{76.9}$ & 70.4 & 79.2 & 81.2 & 74.1 \\
\hline 12 & Mid-patellar height & $43.1-47.2$ & $\underline{43.2}$ & 50.8 & $\underline{46.7}$ & 48.0 & 43.0 & $\underline{43.6}$ & 48.0 & 47.7 & $\underline{45.0}$ \\
\hline 13 & Hip breadth & $31.3-34.3$ & $\underline{31.6}$ & 30.6 & $\underline{32.8}$ & $\underline{33.4}$ & $\underline{32.5}$ & 29.7 & 31.9 & $\underline{33.9}$ & $\underline{32.1}$ \\
\hline 14 & Bicristal breadth & $25.9-29.0$ & $\underline{\underline{26.9}}$ & 25.8 & $\underline{\underline{26.1}}$ & $\underline{28.7}$ & 25.7 & 21.7 & 23.1 & 24.3 & 24.8 \\
\hline 15 & Bitrochanteric breadth & $30.5-33.8$ & 29.7 & 29.9 & $\underline{31.5}$ & $\underline{33.0}$ & $\underline{31.3}$ & 28.4 & $\underline{\underline{30.5}}$ & $\underline{31.2}$ & $\underline{30.6}$ \\
\hline 16 & Thigh breadth & $15.3-18.2$ & 14.8 & 12.8 & $\underline{16.2}$ & $\underline{16.6}$ & $\underline{15.4}$ & 14.0 & 15.2 & $\underline{17.8}$ & $\underline{17.2}$ \\
\hline 17 & Knee breadth & $10.2-11.9$ & 9.9 & 10.0 & $\underline{11.3}$ & $\underline{11.7}$ & 9.9 & 9.8 & $\underline{10.7}$ & $\underline{11.8}$ & $\underline{10.7}$ \\
\hline 18 & Maximum lower leg breadth & $10.2-12.1$ & 9.6 & 9.9 & $\underline{10.9}$ & $\underline{11.8}$ & $\underline{10.3}$ & 10.0 & $\underline{11.0}$ & $\underline{12.1}$ & $\underline{11.0}$ \\
\hline 19 & Waist depth & $17.5-21.8$ & 14.4 & 15.4 & $\underline{18.7}$ & $\underline{\underline{20.7}}$ & 15.8 & 16.8 & 16.6 & $\underline{19.7}$ & $\underline{\underline{20.8}}$ \\
\hline 20 & Abdominal depth & $16.8-21.4$ & 15.1 & 16.2 & $\underline{19.3}$ & 21.4 & 15.9 & $\underline{17.6}$ & $\underline{17.7}$ & $\underline{19.2}$ & 21.4 \\
\hline 21 & Abdominal extension depth & $18.7-22.1$ & 15.6 & 16.4 & $\underline{19.4}$ & 22.9 & 16.3 & 18.2 & 18.2 & $\underline{19.9}$ & $\underline{21.8}$ \\
\hline 22 & Buttock depth & $22.0-25.1$ & 18.8 & 17.5 & 21.7 & $\underline{22.1}$ & 19.2 & 18.9 & 18.9 & 21.4 & 21.3 \\
\hline 23 & Thigh depth & $16.2-18.8$ & 15.0 & 14.8 & $\underline{16.7}$ & $\underline{18.1}$ & 15.5 & 15.7 & 15.6 & $\underline{17.8}$ & $\underline{16.8}$ \\
\hline 24 & Knee depth & $11.4-12.7$ & 11.3 & $\underline{11.6}$ & $\underline{12.6}$ & $\underline{12.7}$ & 11.2 & 10.9 & $\underline{11.8}$ & 12.8 & $\underline{11.4}$ \\
\hline 25 & Maximum lower leg depth & $11.0-12.2$ & 9.7 & 10.5 & $\underline{11.4}$ & 12.6 & 10.5 & 10.3 & $\underline{11.5}$ & 12.7 & $\underline{11.4}$ \\
\hline & Number of maching (part) & & 6 & 3 & 21 & 17 & 9 & 4 & 17 & 17 & 20 \\
\hline & Maching ratio (\%) & & 24 & 12 & 84 & 68 & 36 & 16 & 68 & 68 & 80 \\
\hline
\end{tabular}

from each database. As shown in Fig. 2, in this case, the $S B$ was defined as $-S T D_{A I S T} \leq S B \leq+S T D_{H Q L}$.

In Table 1, all the dimensions included in the $S B$ are indicated in boldface font, and the numbers of applicable parts have been counted. From the results, 21 out of 25 dimensions matched Panel 3 (i.e., 84 \%); hence, Panel 3 was selected. As shown in Fig. 1 (c), the external mold from the upper waist to the lower thigh was produced from the model using gypsum.

\subsubsection{Consideration of motion test}

In a test to assess the motion adaptability of test persons, it is necessary to define an easier, clearer, and specific motion test. This is because the motions that are observed in everyday life are complex, and thus their adaptation as test motions may cause intraand inter-individual variability. The requirements for

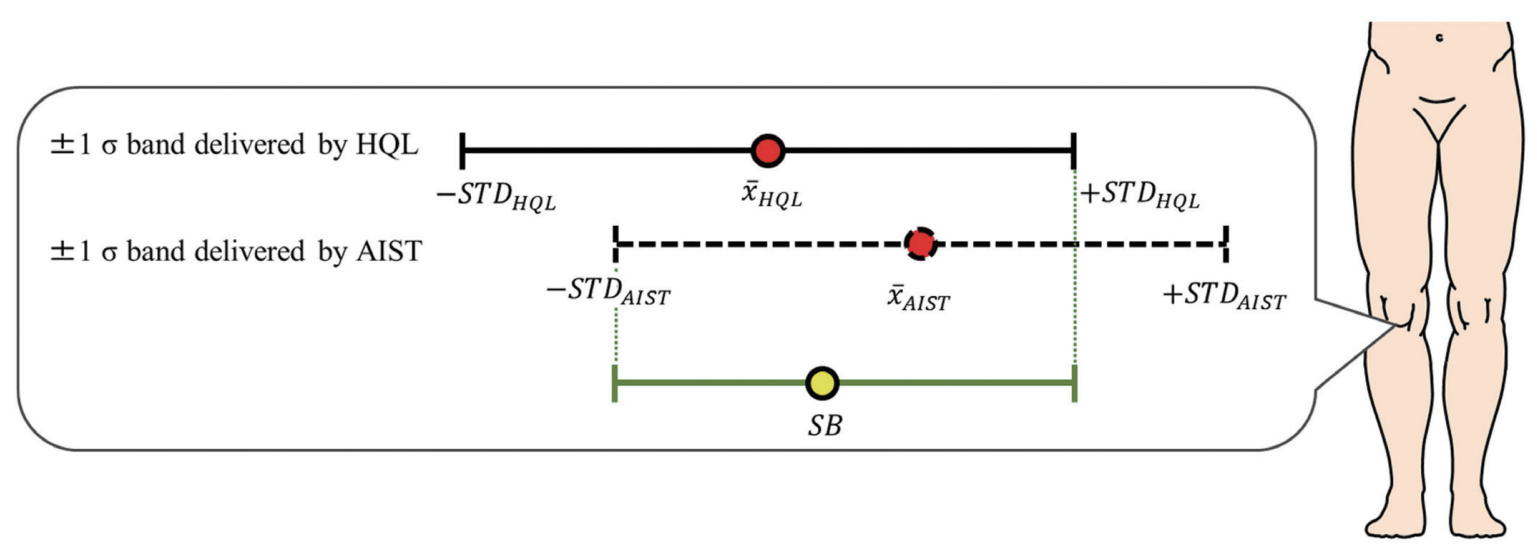

Fig. 2 Definition of standard band $(S B)$ 
the experimental motion were as follows:

- For a substantive evaluation, a motion that is frequently observed in daily life should be assumed (e.g., walking up the stairs).

- To improve the reproducibility of the motion test, a motion conducted using one or two joints in a specific fundamental plane, such as the sagittal, frontal, or horizontal plane, should be selected.

- To simplify data interpretation, a motion that results in a larger clothing deformation should be selected.

Considering the above requirements, the motion test that assumes walking up and down the stairs was adopted in conventional research [16, 18, 19]. As shown in Fig. 1 (b), first, the test person maintained an upright posture as an initial position. Subsequently, the test person elevated his right lower limb in the sagittal plane such that the flexion angle in the knee and hip joints was $90^{\circ}$ (hereinafter, referred to as posture of "one leg raising"). After maintaining this posture, the test person returned his elevated leg to the initial position. The series of the posture changes was defined as the motion test (hereinafter, referred to as "one leg raising").

This study employed the "one leg raising" in the kinematic soft dummy.

\subsubsection{Molding and forming of soft dummy}

Instead of an elaborate skeleton, a mechanical structure that simulated the skeleton of the lower half of the body (hereinafter, referred to as "mechanical skeleton”), as shown in Fig. 1 (c), was inserted into the dummy, and the surrounding dummy model was produced using polyurethane (PU). The rationale behind this practice was that by simplifying the structure of the dummy, its durability could be enhanced and the risk of damage, such as interfacial delamination, could be reduced.

As shown in Fig. 1 (c), the mechanical skeleton consists of joints and shafts simulating the hip joint, knee joint, and bones of the lower limbs. These joints were covered using cylindrical housing with bellows such that the angle could be changed after molding in PU.

PU (Hei-Cast W2870, H\&K Limited) [20] was chosen to reproduce the stiffness of human tissues. The features of this material - low rigidity and high elasticity - are considered suitable for molding humanlike soft tissues. The compression stiffness of PU can be manipulated by selectively varying the proportion of polyol and additives.
The specific PU had a polyol content of $65 \mathrm{wt} \%$ and an additive content of $35 \mathrm{wt} \%$. As shown in Fig. 1 (d), to select the most suitable PU, its stiffness under different conditions was measured and the one that best simulated the stiffness of human tissues was chosen [21].

The soft dummy was produced via the following four steps.

(i) A mechanical skeleton made of stainless steel was fixed in the mold.

(ii) The polyol and additive liquids were mixed in a weight ratio of $65: 35$ in a container.

(iii) The liquid mixture was carefully poured into the tissue mold.

(iv) PU was dried for $24 \mathrm{~h}$ until it was solidified.

After the PU molded soft dummy was developed, the kinematic function was integrated through a control system. In other words, the backside of the right foot of the soft dummy was fixed on a slider such that it could slide along one axis on an automatic stage (IKO LWFHI60, Nippon Thompson Co., Ltd.) [22]. This system enabled the conducting of the "one leg raising" on the dummy of the lower half of the body, as shown in Fig. 1 (e).

A flexion angle $(\alpha)$ in the hip joints of the kinematic dummy was controlled through a migration length (d) of the slider; for example, when $\mathrm{d}=537 \mathrm{~mm}$, $\alpha=90^{\circ}$. Further, when the maximum migration length $\mathrm{d}_{\max }=611 \mathrm{~mm}$, the maximum flection angle $\alpha_{\max }=98.5^{\circ}$. The controllable range of a migration velocity was from 9 to $269 \mathrm{~mm} / \mathrm{s}$. This velocity required $60-2$ s to rotate $\alpha$ from 0 to $90^{\circ}$ in "one leg raising." It was possible to set a repeating number of the "one leg raising" from 1 to 9999.

\subsubsection{Validation of dimensions and stiffness}

The dimensions, stiffness, and kinematics of the kinematic soft dummy were validated.

(1) The dimensions of the circumference of the waist, hip, thigh, and knee were directly measured according to the Martin method [17].

(2) The stiffness of the kinematic soft dummy was investigated. Ito et al. reported that when the stiffness at the location of the sensor is lower, the clothing pressure increases owing to the stress concentration [23]. Thus, the stiffness of the kinematic dummy was compared with that of the test persons. Eleven Japanese male students in their $20 \mathrm{~s}$ were recruited as test persons. Here, the apparent stiffness, including viscosity and elasticity, was measured by applying the press-in method with a durometer-type muscular 
stiffness meter (NEUTONE TDM-N 1, TRY-ALL) [24]. In other words, the elastic force generated was measured by pushing an indenter of the instrument into the test persons or kinematic soft dummy under a force of $14.71 \mathrm{~N}$. The pressed-in force was set at an appropriate magnitude to detect the influence of the contraction/relaxation of muscles located at the shallow layer (e.g., trapezius muscle, erector spinae muscle, or soleus muscle). This study focused on the stiffness including the influence of the contraction/ relaxation of muscles located at the buttock and thigh.

The stiffness was measured at eight locations where the clothing pressure was measured, as shown in Fig. 3 (ch1-ch8). Specifically, there were two points at the knee (ch1: center of patella bone, ch2: upper patella bone), two points at the front of the thigh (ch3: $120 \mathrm{~mm}$ above ch2, ch4: $80 \mathrm{~mm}$ below the inguinalis), two points in the inner thigh (ch5: at the point 3/8 from the knee joint to the inguinalis, ch6: at the point $3 / 4$ from the knee joint to the inguinalis), one point at the back of the thigh (ch7: $100 \mathrm{~mm}$ below the point of maximum width of the thigh girth), and one point at the buttock (ch8: $40 \mathrm{~mm}$ below the peak of the buttock). The circled numbers in the figure represent measurement channels (e.g. 1 represents ch1). During the measurement, the posture was "one leg raising." The measurement was conducted seven times at each channel.

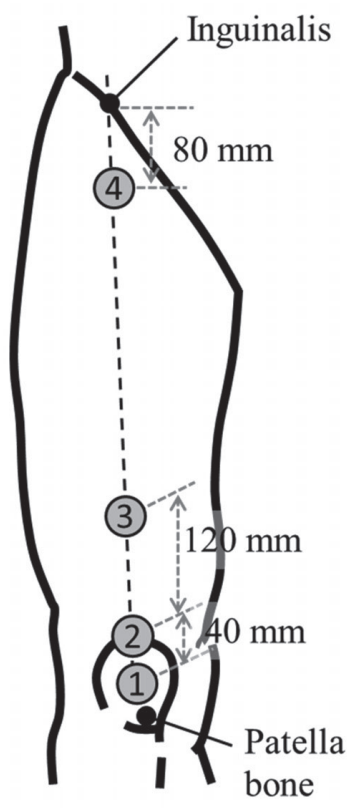

(a)

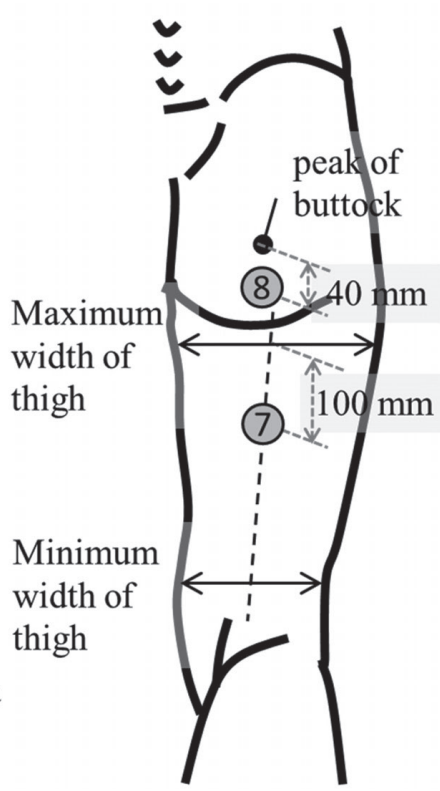

(b)

\subsection{Measurement of clothing pressure of kinematic soft dummy and test persons \\ 2.2.1 Samples}

The men's suit pants produced experimentally through the mechanical stretch method in conventional research were used as samples, as listed in Table 2 [16].

The mechanical and surface properties of the fabric samples were assessed using a KES-FB system (Kato Tech. Co.) under standard conditions [25]. The abbreviations of the parameters discussed in this study are presented in Table 3 .

From the produced fabrics, specimens of $200 \times$ $200 \mathrm{~mm}$ were sampled and tested under warp and weft conditions. Prior to the measurement, the specimens were maintained for $24 \mathrm{~h}$ at a temperature of $23{ }^{\circ} \mathrm{C}$ and relative humidity of $50 \%$. In compliance with ISO 139:2005 [26], the measurements were conducted at a temperature of $23.0 \pm 0.8{ }^{\circ} \mathrm{C}$ and relative humidity of $48 \pm 3 \%$.

The mean values for each parameter are listed in Table 4(b). The value of the EMT in the weft condition changed from $5.9 \%$ to $16.1 \%$ gradually; however, it was approximately the same in the warp condition. The fabric with a larger EMT in the weft condition also had a larger tensile energy because of the larger strain that occurred when the maximum load $(=50 \mathrm{~N} / \mathrm{m})$ acted on the fabric samples. The

Fig. 3 Locations of stiffness validation on test persons and kinematic soft dummy: (a) Front view, (b) back view, and (c) inside view, where 1: center of patella bone, 2: upper patella bone, 3: $120 \mathrm{~mm}$ above ch2, 4: $80 \mathrm{~mm}$ below the inguinalis, 5 : at the point $3 / 8$ from the knee joint to the inguinalis, 6 : at the point $3 / 4$ from the knee joint to the inguinalis, 7: $100 \mathrm{~mm}$ below the maximum width of the thigh girth, and 8: $40 \mathrm{~mm}$ below the peak of the buttock. 
Table 2 Fundamental specification of woven fabric: specifications after finishing process, where $\mu$ : mean value and $\sigma$ : standard deviation.

\begin{tabular}{|c|c|c|c|c|c|c|c|c|}
\hline \multirow{2}{*}{ Sample } & \multirow{2}{*}{ Weave } & & \multicolumn{2}{|c|}{ Apparent yarn count (dtex) } & \multicolumn{2}{|c|}{ Yarn density $(\mathrm{N} . / 2.54 \mathrm{~cm})$} & \multicolumn{2}{|c|}{ Mass per unit Thickness } \\
\hline & & & Warp & Weft & Warp & Weft & area $\left(\mathrm{g} / \mathrm{m}^{2}\right)$ & $(\mathrm{mm})$ \\
\hline \multirow{2}{*}{ S1 } & \multirow{2}{*}{ Plain } & $\mu$ & $137 \times 2$ & $141 \times 2$ & 64.0 & 58.9 & 144.8 & 0.39 \\
\hline & & $\sigma$ & 3 & 5 & 0.8 & 2.0 & 0.0 & 0.01 \\
\hline \multirow{2}{*}{ S2 } & \multirow{2}{*}{ Plain } & $\mu$ & $138 \times 2$ & $135 \times 2$ & 64.8 & 58.7 & 136.3 & 0.35 \\
\hline & & $\sigma$ & 2 & 2 & 1.3 & 1.3 & 0.0 & 0.01 \\
\hline \multirow{2}{*}{ S3 } & \multirow{2}{*}{ Plain } & $\mu$ & $132 \times 2$ & $142 \times 2$ & 64.3 & 57.9 & 140.8 & 0.35 \\
\hline & & $\sigma$ & 2 & 1 & 0.5 & 1.9 & 2.1 & 0.00 \\
\hline \multirow{2}{*}{ S4 } & \multirow{2}{*}{ Plain } & $\mu$ & $138 \times 2$ & $138 \times 2$ & 64.5 & 58.9 & 143.6 & 0.38 \\
\hline & & $\sigma$ & 2 & 2 & 2.1 & 3.0 & 1.8 & 0.01 \\
\hline \multirow{2}{*}{ S5 } & \multirow{2}{*}{ Plain } & $\mu$ & $135 \times 2$ & $140 \times 2$ & 66.5 & 58.7 & 149.5 & 0.40 \\
\hline & & $\sigma$ & 2 & 2 & 0.6 & 1.0 & 0.3 & 0.00 \\
\hline \multirow{2}{*}{ S6 } & \multirow{2}{*}{ Plain } & $\mu$ & $135 \times 2$ & $144 \times 2$ & 67.3 & 57.9 & 150.1 & 0.39 \\
\hline & & $\sigma$ & 4 & 3 & 0.5 & 0.8 & 0.9 & 0.01 \\
\hline
\end{tabular}

Table 3 Mechanical properties of woven fabrics: four parameters related to the tensile property (i.e., LT: linearity, WT: tensile energy, RT: resilience, and EMT: elongation), two parameters related to the bending property (i.e., B: bending rigidity, and $2 \mathrm{HB}$ : hysteresis of bending moment), three parameters related to the shear property (i.e., G: shear stiffness, $2 \mathrm{HG}$ : hysteresis of shear force at $0.5^{\circ}$, and 2 HG 5 : hysteresis of shear force at $5^{\circ}$ ), three parameters related to the surface property (i.e., MIU: friction coefficient, MMD: mean deviation of MIU, and SMD: geometrical roughness), three parameters related to the compression property (i.e., LC: linearity, WC: compressional energy, and RC: resilience).

\begin{tabular}{|c|c|c|c|c|c|c|c|c|c|c|c|c|c|c|c|c|}
\hline \multirow{2}{*}{\multicolumn{2}{|c|}{ Sample }} & \multicolumn{4}{|c|}{ Tensile } & \multicolumn{3}{|c|}{ Shear } & \multicolumn{2}{|c|}{ Bending } & \multicolumn{3}{|c|}{ Surface } & \multicolumn{3}{|c|}{ Compression } \\
\hline & & $\begin{array}{l}\text { LT } \\
(-)\end{array}$ & $\begin{array}{c}\text { WT } \\
\left(\mathrm{gf} \cdot \mathrm{cm} / \mathrm{cm}^{2}\right)\end{array}$ & $\begin{array}{l}\mathrm{RT} \\
(\%)\end{array}$ & $\begin{array}{l}\text { EMT } \\
(\%)\end{array}$ & $\underset{(\mathrm{g} f / \mathrm{cm} \cdot \mathrm{deg})}{\mathrm{G}}$ & $\begin{array}{c}2 \mathrm{HG} \\
(\mathrm{g} / \mathrm{cm})\end{array}$ & $\begin{array}{l}2 \mathrm{HGS} 5 \\
(\mathrm{~g} / \mathrm{cm})\end{array}$ & $\begin{array}{c}\mathrm{B} \\
\left(\mathrm{gf} \cdot \mathrm{cm}^{2} / \mathrm{cm}\right)\end{array}$ & $\begin{array}{c}2 \mathrm{HB} \\
(\mathrm{gf} \cdot \mathrm{cm} / \mathrm{cm})\end{array}$ & $\begin{array}{l}\text { MIU } \\
(-)\end{array}$ & $\begin{array}{l}\text { MMD } \\
(-)\end{array}$ & $\begin{array}{l}\mathrm{SMD} \\
(\mu \mathrm{m})\end{array}$ & $\begin{array}{l}\mathrm{LC} \\
(-)\end{array}$ & $\begin{array}{c}\text { WC } \\
\left(\mathrm{gf} \cdot \mathrm{cm} / \mathrm{cm}^{2}\right)\end{array}$ & $\begin{array}{l}\mathrm{RC} \\
(\%)\end{array}$ \\
\hline \multirow{2}{*}{ s1 } & warp & 0.66 & 5.35 & 87.9 & 3.3 & 1.06 & 0.84 & 1.81 & 0.065 & 0.023 & 0.152 & 0.027 & 3.46 & \multirow{2}{*}{0.287} & \multirow{2}{*}{0.063} & \multirow{2}{*}{70.0} \\
\hline & weft & 0.63 & 9.21 & 80.2 & 5.9 & 0.99 & 0.78 & 1.69 & 0.054 & 0.017 & 0.157 & 0.019 & 3.28 & & & \\
\hline \multirow{2}{*}{ s2 } & warp & 0.65 & 4.87 & 89.1 & 3.0 & 0.83 & 0.59 & 1.22 & 0.061 & 0.019 & 0.164 & 0.027 & 3.22 & \multirow{2}{*}{0.355} & \multirow{2}{*}{0.055} & \multirow{2}{*}{71.8} \\
\hline & weft & 0.58 & 10.89 & 79.1 & 7.5 & 0.79 & 0.58 & 1.20 & 0.048 & 0.012 & 0.172 & 0.025 & 3.59 & & & \\
\hline \multirow{2}{*}{ s3 } & warp & 0.70 & 4.84 & 87.6 & 2.8 & 0.78 & 0.72 & 1.64 & 0.074 & 0.029 & 0.164 & 0.017 & 2.74 & \multirow{2}{*}{0.360} & \multirow{2}{*}{0.064} & \multirow{2}{*}{62.8} \\
\hline & weft & 0.63 & 14.01 & 74.3 & 9.0 & 0.72 & 0.77 & 1.66 & 0.052 & 0.019 & 0.138 & 0.016 & 2.69 & & & \\
\hline \multirow{2}{*}{ s4 } & warp & 0.66 & 4.87 & 88.1 & 3.0 & 0.88 & 0.63 & 1.37 & 0.062 & 0.021 & 0.187 & 0.025 & 2.45 & \multirow{2}{*}{0.347} & \multirow{2}{*}{0.064} & \multirow{2}{*}{65.3} \\
\hline & weft & 0.59 & 15.90 & 73.3 & 10.7 & 0.79 & 0.71 & 1.33 & 0.042 & 0.012 & 0.163 & 0.020 & 2.32 & & & \\
\hline \multirow{2}{*}{ s5 } & warp & 0.62 & 5.57 & 87.1 & 3.6 & 0.90 & 0.65 & 1.50 & 0.065 & 0.020 & 0.178 & 0.017 & 2.24 & \multirow{2}{*}{0.284} & \multirow{2}{*}{0.064} & \multirow{2}{*}{59.7} \\
\hline & weft & 0.59 & 18.29 & 71.1 & 12.4 & 0.82 & 0.82 & 1.55 & 0.037 & 0.013 & 0.145 & 0.018 & 2.80 & & & \\
\hline \multirow{2}{*}{ s6 } & warp & 0.73 & 5.40 & 87.0 & 2.9 & 0.75 & 0.68 & 1.49 & 0.070 & 0.026 & 0.219 & 0.026 & 3.20 & \multirow{2}{*}{0.327} & \multirow{2}{*}{0.070} & \multirow{2}{*}{60.6} \\
\hline & weft & 0.62 & 24.92 & 63.8 & 16.1 & 0.63 & 0.74 & 1.43 & 0.027 & 0.012 & 0.168 & 0.017 & 2.32 & & & \\
\hline
\end{tabular}

Table 4 Dimensions of sample pants and dimensions of evaluation panel

\begin{tabular}{cccc}
\hline \multicolumn{4}{c}{ (a) Circumference of sample (cm) } \\
Waist & Hip & Thigh & Knee \\
\hline 70 & 89.0 & 62.0 & 44.0 \\
\hline 73 & 91.9 & 63.2 & 44.8 \\
\hline 76 & 94.8 & 64.4 & 45.6 \\
\hline 85 & 103.5 & 68.0 & 49.0 \\
\hline 73 & 91.9 & 63.2 & 44.8 \\
\hline 70 & 89.0 & 62.0 & 44.0 \\
\hline 76 & 94.8 & 64.4 & 45.6 \\
\hline 79 & 97.7 & 65.6 & 46.4 \\
\hline 82 & 100.6 & 66.8 & 47.2 \\
\hline 82 & 100.6 & 66.8 & 47.2 \\
\hline 76 & 94.8 & 64.4 & 45.6 \\
\hline 73 & 91.9 & 63.2 & 44.8 \\
\hline \hline
\end{tabular}

\begin{tabular}{cccc}
\hline \multicolumn{4}{c}{ (b) Circumference of body $(\mathrm{cm})$} \\
Waist & Hip & Thigh & Knee \\
\hline 65.2 & 87.0 & 48.9 & 34.8 \\
\hline 65.5 & 84.2 & 46.5 & 35.0 \\
\hline 76.2 & 91.5 & 53.3 & 38.3 \\
\hline 79.5 & 98.8 & 59.2 & 40.1 \\
\hline 67.5 & 89.5 & 50.8 & 34.4 \\
\hline 66.5 & 84.2 & 47.7 & 34.8 \\
\hline 72.0 & 90.5 & 52.9 & 36.4 \\
\hline 75.6 & 93.6 & 57.5 & 38.6 \\
\hline 80.5 & 91.2 & 55.3 & 34.5 \\
\hline 76.6 & 90.8 & 54.5 & 35.1 \\
\hline 72.8 & 90.8 & 53 & 36.9 \\
\hline 68.9 & 84.0 & 47.1 & 35.7 \\
\hline \hline
\end{tabular}

\begin{tabular}{cccc}
\hline \multicolumn{4}{c}{ (c) Difference ( (a) - (b)) (cm) } \\
Waist & Hip & Thigh & Knee \\
\hline 4.8 & 2.0 & 13.1 & 9.2 \\
\hline 7.5 & 7.7 & 16.7 & 9.8 \\
\hline-0.2 & 3.3 & 11.1 & 7.3 \\
\hline 5.5 & 4.7 & 8.8 & 8.9 \\
\hline 5.5 & 2.4 & 12.4 & 10.4 \\
\hline 3.5 & 4.8 & 14.3 & 9.2 \\
\hline 4.0 & 4.3 & 11.5 & 9.2 \\
\hline 3.4 & 4.1 & 8.1 & 7.8 \\
\hline 1.5 & 9.4 & 11.5 & 12.7 \\
\hline 5.4 & 9.8 & 12.3 & 12.1 \\
\hline 3.2 & 4.0 & 11.4 & 8.7 \\
\hline 4.1 & 7.9 & 16.1 & 9.1 \\
\hline \hline
\end{tabular}

\begin{tabular}{cccc}
\hline \hline \multicolumn{4}{c}{ (d) Proportion $((\mathrm{a}) /(\mathrm{b}) \times 100)(\%)$} \\
Waist & Hip & Thigh & Knee \\
\hline 6.9 & 2.2 & 21.1 & 20.9 \\
\hline 10.3 & 8.4 & 26.4 & 21.9 \\
\hline-0.3 & 3.5 & 17.2 & 16.0 \\
\hline 6.5 & 4.5 & 12.9 & 18.2 \\
\hline 7.5 & 2.6 & 19.6 & 23.2 \\
\hline 5.0 & 5.4 & 23.1 & 20.9 \\
\hline 5.3 & 4.5 & 17.9 & 20.2 \\
\hline 4.3 & 4.2 & 12.3 & 16.8 \\
\hline 1.8 & 9.3 & 17.2 & 26.9 \\
\hline 6.6 & 9.7 & 18.4 & 25.6 \\
\hline 4.2 & 4.2 & 17.7 & 19.1 \\
\hline 5.6 & 8.6 & 25.5 & 20.3 \\
\hline \hline
\end{tabular}


fabric with a larger EMT in the weft condition also had a smaller resilience. Thus, the recovery was inferior for the sample with superior elongation. However, no apparent relationship or tendency was detected between the EMT and other parameters of the shearing, bending, surface, and compression properties in the weft and warp conditions.

The men's suit pants consisted of worsted fabrics with different EMTs but a similar fundamental structural specification, such as yarn count, yarn density, and weaving.

Six different suit pants were produced from the fabric samples by a technician trained in sewing, using a specific pattern. The commercialized pattern was provided by Aoki Holdings Co. Ltd. All necessary sizes of the suit pants were produced and were adapted to the body sizes of the panels.

In this study, the pants were produced from six worsted fabric samples with different stretchability in the weft direction. The six sample pants were produced in six different sizes, i.e., a waist circumference of $70 \mathrm{~cm}$ with increments of $3 \mathrm{~cm}$ up to $85 \mathrm{~cm}$ (i.e., Sizes 1-6). The dimensions of the waist circumference, hip circumference, thigh circumference, and knee circumference are listed in Table 4.

\subsubsection{Experimental procedure}

The clothing pressure acts on the body as a result of the fabric tension occurring according to the motion. Here, the clothing pressure was measured for the kinematic soft dummy and test persons. The 11 test persons, who participated in the stiffness measurement, were recruited as test persons for the clothing pressure measurement. The dimensions of the waist circumference, hip circumference, thigh circumference and knee circumference are listed in Table 4 . The results of the clothing pressure with the test persons were obtained from existing literature [16].

The clothing pressure of the kinematic soft dummy was measured using the following protocol: (a) the experimenter placed the sample on the kinematic soft dummy appropriately, (b) the kinematic soft dummy was maintained in an upright posture as the initial position, (c) then, its right foot was made to slide along the slider with a velocity of $269 \mathrm{~mm} / \mathrm{s}$ such that it could complete "one leg raising motion" in $3 \mathrm{~s}$ (the required time differed from the case of test persons to prevent damaging the PU. According to our investigation conducted prior to the measurement, this difference in measurement did not influence the results.), and (d) its right lower limb was maintained static such that the flexion/extension angle in the knee/hip joint was $90^{\circ}$ for $11 \mathrm{~s}$.

The clothing pressure of the test persons was measured using the following protocol: the model (a) wore a dress shirt in the upper body, underwear in the lower body, and the sample was offered by the experimenter with a belt, (b) maintained an upright posture as an initial position, (c) conducted the "one leg raising" in $2 \mathrm{~s}$, and (d) maintained the right lower limb static such that the flexion/extension angle in the knee/hip joint was $90^{\circ}$ for $12 \mathrm{~s}$. In addition, the test persons were not permitted to breathe during the clothing pressure measurements. Measurements were performed five times for each sample.

The sensor positions were shared in both measurements. In other words, the air-pack sensors (20 mm in diameter, AMI 3037, AMI Co. Ltd.) were placed at eight locations where stiffness was validated at the right half of the body, as shown in Fig. 3 (ch1ch8). The maximum thickness of the sensor was $1 \mathrm{~mm}$, and the sampling frequency was $2.5 \mathrm{~Hz}$.

The order in which the samples were measured was random.

\subsection{Subjective evaluation of motion adaptability of men's suit pants}

Kanai et al. sensorially evaluated the motion adaptability of men's suit pants in conventional research [16]. In this research, the live panels assessed certain adjectives. In this paper, we focused on and extracted the mean preference score on "Easy to move" and utilized it in a later investigation.

An overview of the sensory evaluation is as follows. This sensory evaluation was conducted using Scheffe's paired comparison method (Nakaya's law) [28]. Eleven Japanese male students in their $20 \mathrm{~s}$ were recruited as the evaluation panels. The panels wore a sample pair of pants labeled either A or B and evaluated which sample was superior to the other one based on particular features through their sensation. Specifically, the panels first wore a pant sample labeled $\mathrm{A}$ and conducted the one leg raising motion. Afterward, they wore the other pant sample labeled $\mathrm{B}$ and conducted the one leg raising motion again. Finally, the panels were instructed to compare A and $\mathrm{B}$ and evaluate them on the provided adjectives. This evaluation process was repeated for the other pairs.

The evaluation was conducted using the following protocol: (a) the live model wore sample A, 
and the experimenter confirmed the wearing status as appropriate, (b) the live model maintained an upright posture as an initial position, (c) conducted the "one leg raising motion" in $2 \mathrm{~s}$, and (d) maintained the right lower limb static so that the flexion/extension angle in the knee/hip joint was $90^{\circ}$ for $5 \mathrm{~s}$, (e) returned the right lower limb to the initial position in $2 \mathrm{~s}$, (f) and replaced sample A with sample B, and the experimenter confirmed the wearing status as appropriate; $(\mathrm{g})$ the live model repeated the above procedure from (b) to (e). Finally, the live model compared samples A and B using the provided adjectives and filled the results in the form.

In addition, to conduct the "one leg raising motion" correctly (especially to control the flexion angle at knee/hip joint accurately), the video was shot by a camera set up at the right side of the panel and was projected simultaneously onto the monitor set up in front of the panel.

The evaluation was conducted using seven scale indicators and transformed into numeral scores. The correspondence between the scores and their meaning was as follows: -3 point is "Sample A was extremely superior," -2 point is "Sample A was moderately superior," -1 point is "Sample A was slightly superior," 0 point is "Neither," +1 point is "Sample B was slightly superior," +2 point is "Sample B was moderately superior," and +3 point is "Sample B was extremely superior."

The human subjects wore a dress shirt in the upper body and underwear in the lower body; then, they wore each sample with a belt. To be compliant with ISO 139:2005 [26], the experimental room was maintained at a temperature of $22.8 \pm 0.4{ }^{\circ} \mathrm{C}$ and a relative humidity of $52 \pm 2 \%$, and the samples were controlled in this environment for $24 \mathrm{~h}$ before conducting the experiment.

\section{Results and Discussion}

\subsection{Result of dimensions and stiffness}

As shown in Table 5, each dimension of the kinematic soft dummy approximately corresponded with that of the model.

As shown in Fig. 4, there was a large difference in the averaged stiffness between the test persons and the kinematic soft dummy. The maximum and minimum values were removed from the dataset at each channel. Then, the averaged value among test persons was calculated at each channel. Therefore, the error bars indicated the standard deviation of the test panels. ANOVA was employed for significance investigation.

A gap of more than $0.5 \mathrm{~N}$ was generated at ch1, $\operatorname{ch} 2$, and ch3, because the existence of the patella bone increased the stiffness on test persons. In other words,

Table 5 Comparison of body dimensions of model with those of kinematic soft dummy, where $\Delta$ corresponds to the difference between the two.

\begin{tabular}{lccc}
\hline & $\begin{array}{c}\text { Test person } \\
\text { (Panel 3) }\end{array}$ & $\begin{array}{c}\text { Kinematic soft } \\
\text { dummy }\end{array}$ & $\Delta$ \\
\hline Waist & 76.2 & 76.0 & -0.2 \\
\hline Hip & 91.5 & 91.0 & -0.5 \\
\hline Thigh & 53.3 & 54.0 & +0.7 \\
\hline Knee & 38.3 & 38.0 & -0.3 \\
\hline
\end{tabular}

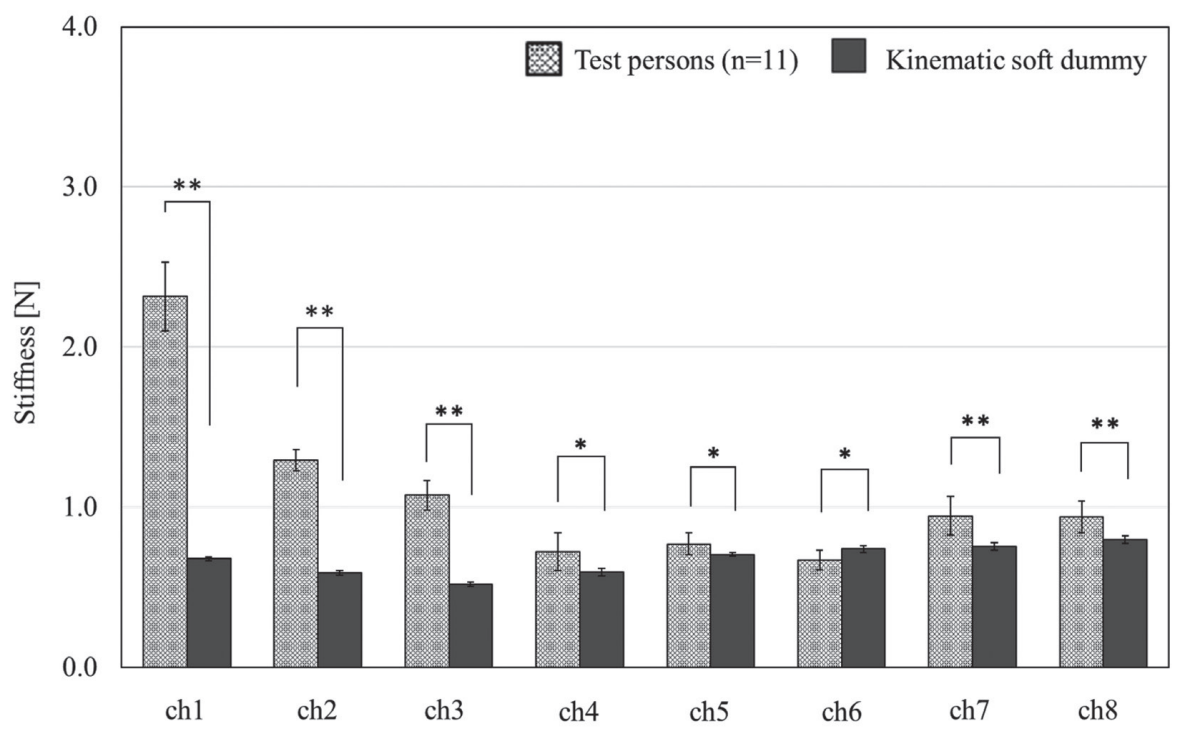

Fig. 4 Comparison of stiffness of test persons $(n=11)$ with that of kinematic soft dummy in "one leg raising" posture: ${ }^{* *} 1 \%$ level of significance and ${ }^{*} 5 \%$ level of significance 
it was considered that the stiffness distribution shown in the test persons was not obtained because of the structural differences at the knee. However, because the gap at ch4, ch5, ch6, ch7, and ch8 was less than $\pm 0.1 \mathrm{~N}$, it was confirmed that the stiffness of the kinematic soft dummy sufficiently simulated that of the test persons.

\subsection{Comparison of clothing pressure value}

The representative time sequence changes of the clothing pressure on Panel 3 for S1 and that on the kinematic soft dummy for S1 are shown in Fig. 5 (a) and (b), respectively. The clothing pressure increased rapidly because of the "one leg raising" motion. After its peak, it decreased gradually and reached approximate equilibrium because of the maintained “one leg raising” posture. In addition, the intensity of clothing pressure varied in individual channels.

From a pretest of this experiment, the standard deviation of the time sequence changes fell under $0.1 \mathrm{kPa}, 5.2 \mathrm{~s}$ after completing the posture of "one leg raising." Thus, an interval from 7.2 to $10.4 \mathrm{~s}$, after the start of the measurements was set as an analysis band, and the mean value of the clothing pressure was calculated.

A comparison of the averaged clothing pressure of the test persons with that of the kinematic soft dummy is depicted in Fig. 6. The channels are shown in Fig. 3.

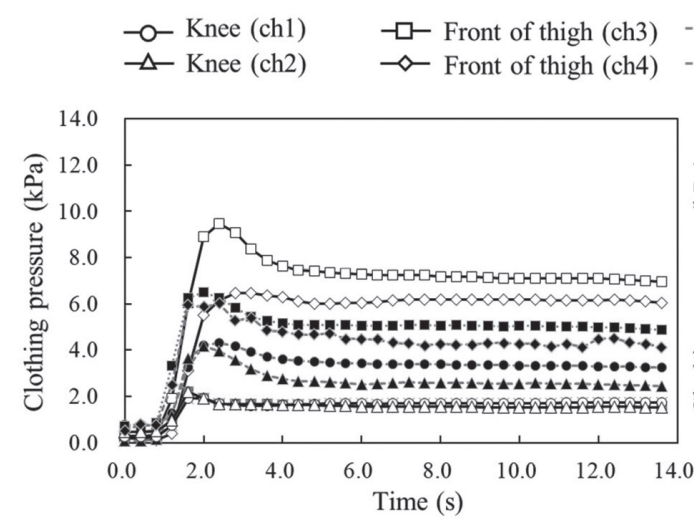

(a)
- Inner thigh (ch5)

Inner thigh (ch6)
-. Back of thigh (ch7)

- Buttock (ch8)

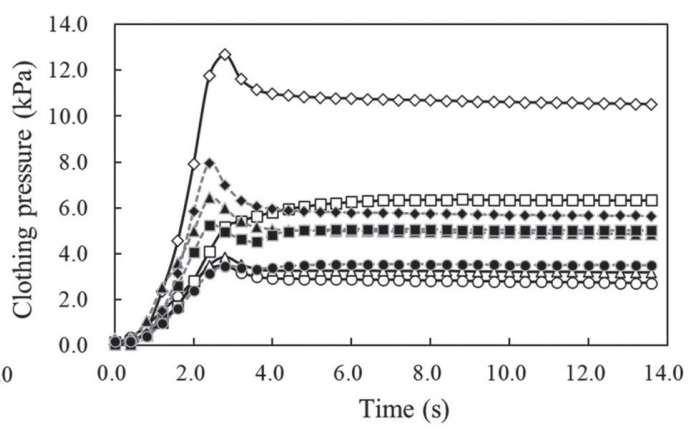

(b)

Fig. 5 Time sequence changes of clothing pressure at different channels: (a) On test person (Panel 3, S1); (b) On kinematic soft dummy (S1)

$\mathrm{S} 1 \square \mathrm{S} 2 \square \mathrm{S} 3 \square \mathrm{S} 4 \square \mathrm{S} 5 \square \mathrm{S} 6$ $* *: 1 \%$ S.L. $*: 5 \%$ S.L.

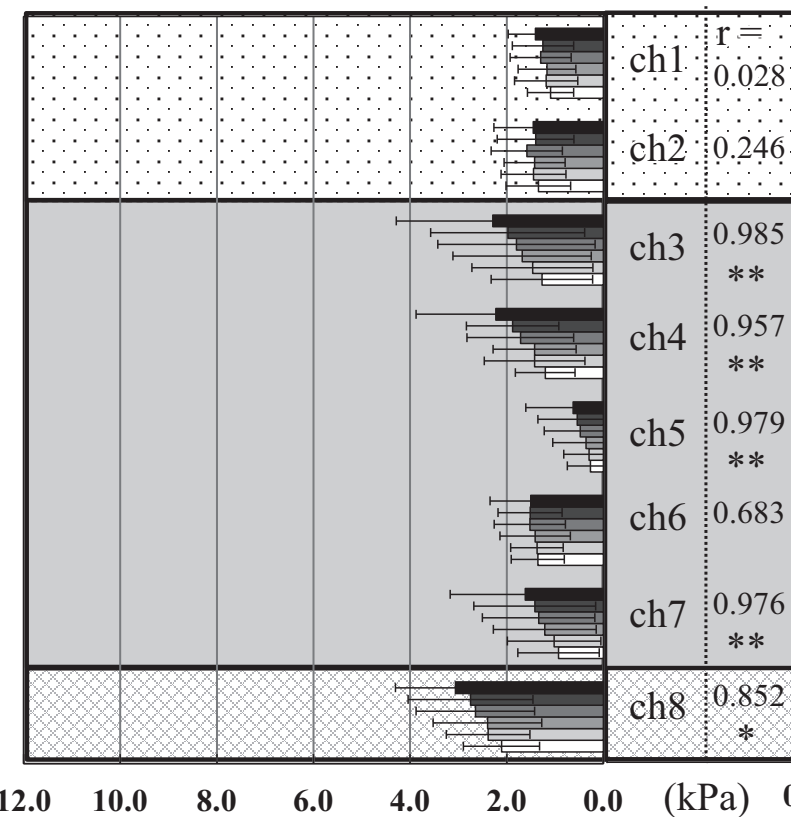

(a) Test persons $(n=11)$
$* *: 1 \%$ S.L. $*$ : $\%$ S.L.

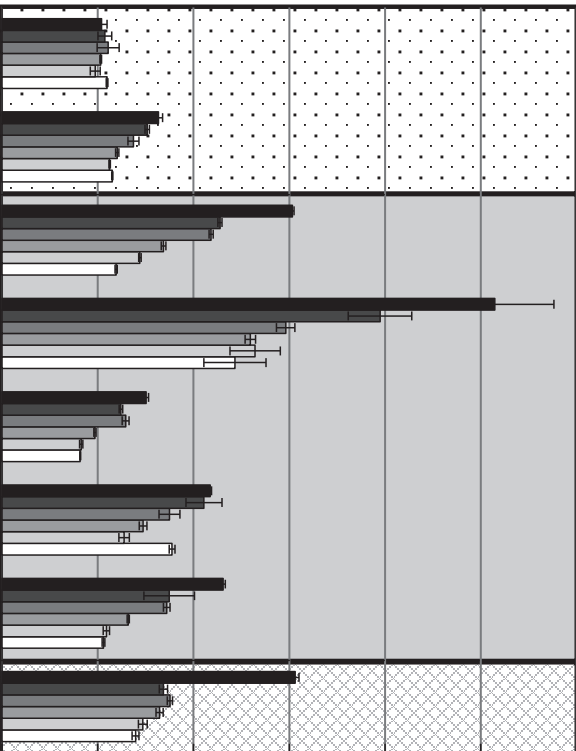

$\begin{array}{llllll}2.0 & 4.0 & 6.0 & 8.0 & 10.0 & 12.0\end{array}$

(b) Kinematic soft dummy

Fig. 6 Result of clothing pressure measurement: (a) Left region indicates clothing pressure and its standard deviation on live panels. (b) Right region indicates that on kinematic soft dummy. Numerals in the center of left region and right region denote the correlation coefficient for the clothing pressure of the live panels and the kinematic soft dummy, where S.L.: significance level., and S1-S6: the six samples. 
In the results of the test persons, the error bars indicate the standard deviation across the test persons, i.e., the issue of individual differences. It was considered that the individual differences were mainly caused by the low agreement of body dimensions and low reproducibility of the motion test. On the other hand, in the results of the kinematic soft dummy, the error bars indicate the standard deviation across the five measurements, i.e., the issue of reproducibility. In comparison, the error bar in the kinematic soft dummy is much smaller than that in the live panels. Thus, a significant decrease of deviation was one of the merits of applying the kinematic soft dummy to the measurement of clothing pressure as an alternative to the test persons.

Tamura et al. mentioned that $1.88 \mathrm{kPa}$ is the level of clothing pressure that causes a reduction in blood flow [23]. In the test persons, the parts on which the clothing pressure had such an effect were the front of the thigh (ch3, ch4) and the buttock (ch8). On the other hand, in the kinematic soft dummy, the clothing pressure had that effect on all the parts, from ch1 to ch8. The partial clothing pressure of the kinematic soft dummy was from 1.4 to 5.3 times larger than that of the test persons depending on the channel. (i.e., 1.7 times (ch1), 1.9 times (ch2), 2.2 times (ch3), 3.9 times (ch4), 5.3 times (ch5), 2.4 times (ch6), 2.4 times (ch7), and 1.4 times (ch8), respectively).

One of the reasons why a larger clothing pressure occurred in the kinematic soft dummy was the difference in friction property between the surface of the kinematic soft dummy and the skin of the test persons. As shown in Fig. 7 (a) and (b), the height of the hemline on the men's suit pants in the test person (Panel 3) was greater than that in the kinematic soft dummy because of the large slippage of garment cloth over skin. Thus, this picture qualitatively reveals that the kinematic soft dummy has a higher friction coefficient than the skin of the test person. It was considered that the large friction coefficient caused high clothing pressure in the kinematic soft dummy. Furthermore, the lower clothing pressure in the inner thigh (ch5) was caused by the undulation of the skin delivered from the contraction and relaxation of the biceps femoris and quadriceps. In other words, the sensor's position in ch5 was arranged on the part of the valley due to undulation. On the other hand, such an undulation was not observed in the kinematic soft dummy (Fig. 7 (c) and (d)). Therefore, the largest difference between the clothing pressure of the test persons and that of the kinematic soft dummy was in the inner thigh (ch5).

The above results imply that we could significantly reduce the deviation of the clothing pressure across the test persons by applying the kinematic soft dummy to the clothing pressure measurement. However, it was difficult to obtain the absolute value of clothing pressure in contrast to the measurement using test persons.

To investigate whether the tendency of clothing pressure was consistent across the samples, the correlation coefficient for the test persons and the kinematic soft dummy was obtained in each channel. The result is denoted in the center of the left and right region, as shown in Fig. 6. The correlation at ch1 and ch2 (knee) was not statistically significant owing to the fact that the stiffness at both ch1 and ch2 was largely different because the kinematic soft dummy did not have a patella bone, as shown in Fig. 4. However, the correlation at ch3 and ch4 (front of the thigh), ch5 (inner thigh), and ch7 (back of the thigh) was statistically significant at the one percent level. Additionally, the correlation at ch8 (buttock) was

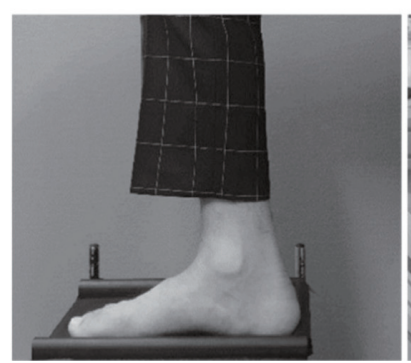

(a)

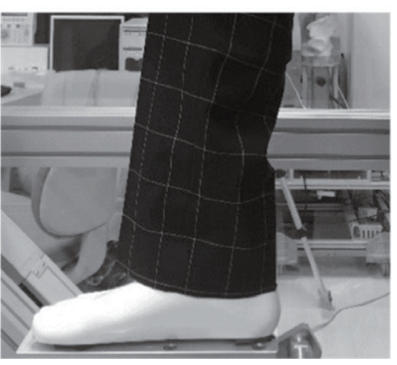

(b)

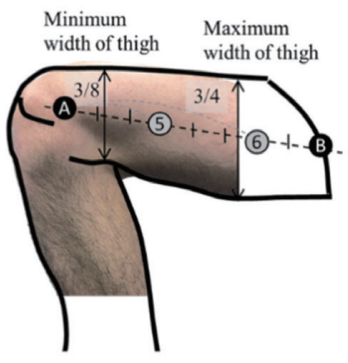

(c)

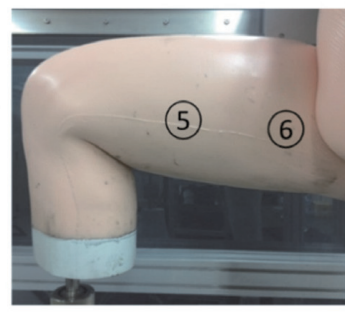

(d)

Fig. 7 Observation of rising hemline of men's suit due to one leg raising motion and observation of undulation in inner thigh: (a) test persons (Panel 3), (b) kinematic soft dummy, (c) test person (Panel 3), (d) kinematic soft dummy, where (5): the point dividing to $3 / 8$ from the knee joint to the inguinalis, and (6): the point dividing to $3 / 4$ from the knee joint to the inguinalis. 


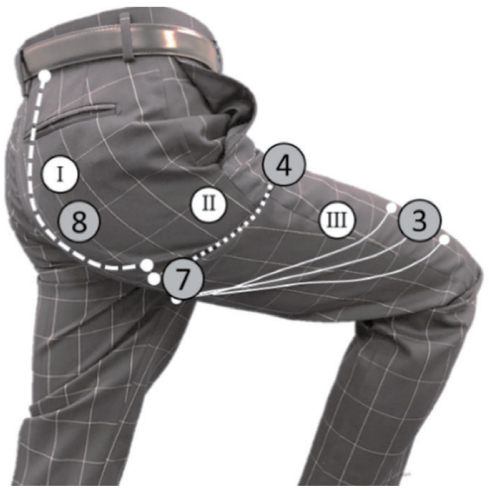

(a)

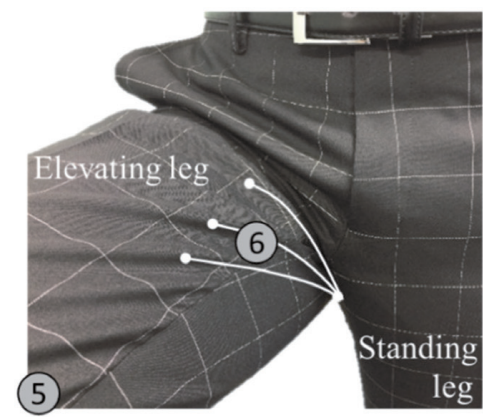

(b)

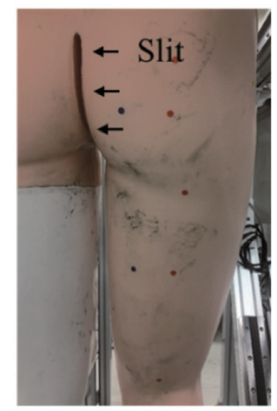

(c)

Fig. 8 Linkage of partial tensile strain and sensor's location: (a) Cloth deformation defined by Kanai et al. (I: Type I, II: Type II, III: Type III) [21], (b) Other cloth deformation, (c) Slit simulating an intergluteal cleft, ( ${ }^{*}$ note: The Roman numeral in white circle denotes the type of cloth deformation; the Albia numeral in gray circle denotes the sensor's location).

statistically significant at the five percent level.

In general, it is well known that clothing pressure results in cloth deformation, such as the large buckling formed from the linkage of a partial tensile strain of fabric [16, 18]. Kanai et al. found three types of cloth deformation (Type I, Type II, and Type III) due to clothing pressure in one leg raising posture, as shown in Fig. 8 (a). Subsequently, they assigned the type of cloth deformation to the location on which clothing pressure acted [16]. In particular, Type I is the cloth deformation due to the partial tensile strain from the belt line to the lower buttock; this causes clothing pressure at ch8 (buttock). Type II is the cloth deformation due to the partial tensile strain from the upper back of the thigh to the upper front of the thigh; this causes the clothing pressure at ch7 (back of the thigh) and ch4 (upper front of the thigh). Type III is the cloth deformation due to the partial tensile strain from the upper back of the thigh to the lower front of the thigh; this causes clothing pressure at ch7 (back of the thigh), ch5 (inner thigh), and ch4 (lower front of the thigh). As described above, the clothing pressures obtained from $\operatorname{ch} 4, \operatorname{ch} 5 \operatorname{ch} 7$, and ch8 were significantly correlated between the kinematic soft dummy and the test persons. Hence, it was considered that the cloth deformation caused in the kinematic soft dummy agreed with that caused in the test persons.

On the other hand, the clothing pressure obtained from ch6 correlated weakly. This was because the cloth deformation caused by clothing pressure at ch6 differed from Type I, Type II, or Type III. It was the cloth deformation due to the partial tensile strain from the back of the thigh on the standing leg (left leg) to the inner thigh on the raised leg (right leg), as shown in Fig. 8 (b). Because the PU elongation at the buttock was less on the kinematic soft dummy than it was on test persons, it was considered that the cloth deformation at the buttock of the standing leg (left leg) was slightly different. To accurately reproduce the cloth deformation at the buttock, an optimization of the design parameters (i.e., length, depth, width, etc.) of a slit simulating an intergluteal cleft of a live model set in the center of the buttock is required (see Fig. 8 (c)). Because the slit can compensate for the lack of elongation on the PU material at the buttock, it may deliver accurate cloth deformation at the buttock.

From the above results, it was found that the clothing pressure at ch3, ch4, ch5, and ch8 measured with the kinematic soft dummy were highly correlated to those measured with test persons. Three types of cloth deformation in the one leg raising motion caused the clothing pressure at these channels. It was considered that the cloth deformation was reproduced accurately in the kinematic soft dummy. In other words, it was confirmed that a reliable dataset could be obtained by measuring the clothing pressure of the kinematic soft dummy.

\subsection{Predictability of motion adaptability from clothing pressure test with kinematic soft dummy}

The mean preference score about "Easy to move" on each sample is shown in Fig. 9. Simultaneously, the result of statistical investigation with a value of yardstick obtained from the analysis process of paired comparison was indicated.

From the comparison of the six pant samples, the mean preference score gradually increased as the EMT in the weft condition increased. It was confirmed that there was a significant difference 


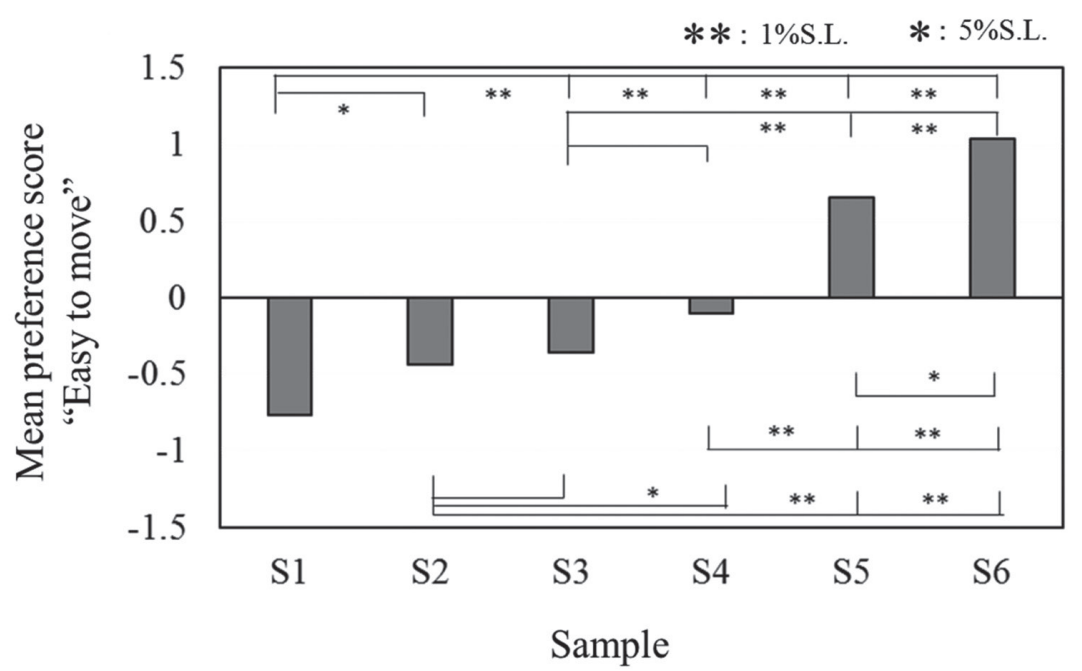

Fig. 9 Mean preference score about "Easy to move” on each sample [16], where S.L.: significance level.

across most of the pairs.

The correlation coefficient for the relationship between the mean preference score about "Easy to move" and the clothing pressure of the kinematic soft dummy at ch3, ch4, ch5, ch7, and ch8 was calculated. These channels were the sensor's position, where a high correlation coefficient for the clothing pressure of the live panels and the kinematic soft dummy was confirmed. The correlation coefficient was as follows: ch3: - 0.925 (1\% significant level (S.L.)), ch4: -0.769 (No significance), ch5: -0.926 (1\% S.L.), ch7: -0.909 (1\% S.L.), and ch8: - 0.711 (No significance).

Subsequently, a single regression analysis was conducted with the mean preference score as a dependent variable and the clothing pressure at ch3, ch5, and ch7 as independent variables. The functions for predicting scores on "Easy to move" (i.e., motion adaptability) were delivered using equations (1), (2), and (3). The relationship between the scores predicted by substituting the clothing pressure into the functions and the mean preference scores evaluated in the sensory test are shown in Fig. 10 (a), (b), and (c). Because all the samples (i.e., S1-S6) were plotted in a region with a $95 \%$ confidence interval (C.I.), it was found that the predicted functions were obtained effectively.

$$
\begin{gathered}
M P S_{M A}=-0.48 C P_{c h 3}+1.90\left(r^{2}=0.928\right)(e q 1) \\
M P S_{M A}=-1.15 C P_{c h 5}+2.56\left(r^{2}=0.930\right)(e q 2) \\
M P S_{M A}=-0.66 C P_{c h 7}+2.03\left(r^{2}=0.913\right)(e q 3) \\
M P S_{M A}: \text { Mean Preference Score on Motion Adaptability } \\
C P_{c h 3}: \text { Clothing Pressure at the front of the thigh } \\
C P_{c h 5}: \text { Clothing Pressure at the inner thigh } \\
C P_{c h 7}: \text { Clothing Pressure at the back of the thigh }
\end{gathered}
$$

From the above results, it was possible to predict the score of motion adaptability of men's suit pants by measuring clothing pressure at ch3, ch5, and ch7 with the kinematic soft dummy.

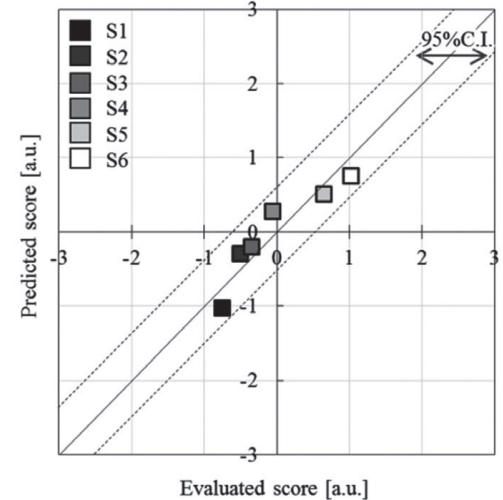

(a)

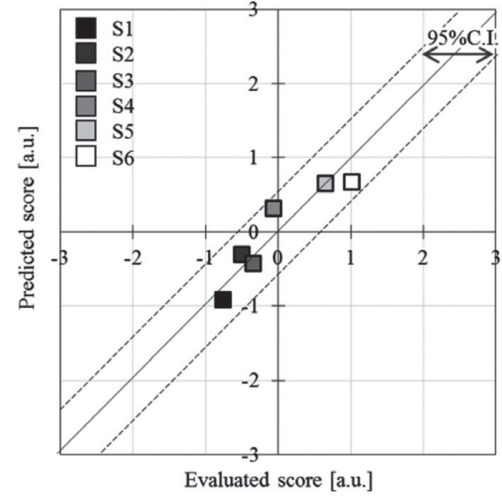

(b)

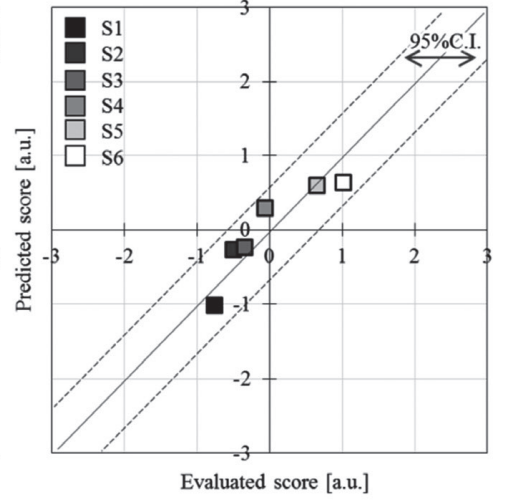

(c)

Fig. 10 Relationship between predicted score and evaluated score: (a) result calculated with equation (1), (b) result calculated with equation (2), (c) result calculated with equation (3), where C.I.: confidence interval and S1-S 6: the six samples. 


\section{Conclusions}

In this study, we designed and developed an anthropomorphic dummy simulating the stiffness of a test person and implemented the kinematic function. Subsequently, we measured the clothing pressure of the kinematic soft dummy with men's suit pants that consisted of six worsted fabrics with an elongation that gradually varied along the weft yarn direction of the fabric from $5.9 \%$ to $16.1 \%$.

For the result of clothing pressure, it was possible to accurately reproduce the specific cloth deformation caused by the one leg raising motion with the kinematic soft dummy. At the locations on which the clothing pressure acted because of the specific cloth deformation, a high correlation coefficient for the clothing pressure of the test person and the kinematic soft dummy was confirmed. In addition, it was possible to significantly reduce the deviation of the clothing pressure across the test persons by applying the kinematic soft dummy to the clothing pressure measurement. Thus, it was confirmed that a reliable dataset could be obtained by measuring the clothing pressure of the kinematic soft dummy.

Furthermore, we investigated the possibility of predicting the score on the motion adaptability of samples that was evaluated by test person $\mathrm{s}$ from the clothing pressure measured with the kinematic soft dummy. The regression equation could deliver accurate predictions by using the clothing pressure measured at ch3, ch5, or ch7. Thus, it was confirmed that the kinematic soft dummy was an effective tool for evaluating the motion adaptability of men's suit pants.

Moreover, more effort is required to achieve higher accuracy. Specifically, the stiffness of the knee should be reproduced. The PU surface should be reformed so that the friction property could be similar to that of the skin of the test person. In addition, to accurately reproduce the cloth deformation at the buttock, an optimization of the design parameter of a slit simulating an intergluteal cleft of a test person set in the center of the buttock is required.

To promote effectiveness and convenience as an evaluation tool, we also started to dynamically measure the clothing pressure using the kinematic soft dummy.

\section{Acknowledgement}

The authors disclose receipt of the following financial support for a Grant-in-Aid for Scientific Research Program by the Ministry of Education, Science, Sports, and Culture of Japan (grant number 20K02343, 20H0028814).

\section{References}

1. S. Jing and T. Tamura, Jpn. J. Clothing Res. 60, 67 (2017).

2. Z. Liu, X. Zhang and S. Hu, J Tex Res China, 27, 104 (2006).

3. H. Fujii, Jpn Res Assn Text End-Uses. 39, 148 (1998).

4. H. Sugaya, K. Iwasaki and Y. Takeda, Investigation of measurement of carrying pressure (translation from Japanese). Report, Tokyo Metropolitan Industrial Technology Research Institute, Japan, September 2015.

5. K. Kazama, K. Ohno, M. Umegae, et al. Dress form of cellular double layer of thermoplastic materials. Patent 3865284, USA, 1975.

6. Y. Shikinami and K. Tsuta, Soft mannequin with gel layers (translation from Japanese). Patent 3806814, Japan, 1999.

7. Y. Shikinami and K. Tsuta. Elastic gel for mannequin (translation from Japanese). Patent 3614316, Japan, 1999.

8. M. Akiyama, Forming skin layer of mannequin (translation from Japanese). Patent 1732055, Japan, 1992.

9. A. Steven, Female functional mannequin. Patent 5466235 A, USA, 1995.

10. W. Yu, J. Fan, X. Qian, et al., J Fiber Sci Tech, 60, 57 (2004).

11. J. Fan, Thermal manikins and modelling. Report, The Hong Kong Polytechnic University, Hong Kong, October 2006.

12. T. Tamura, Development of a movable soft manikin of the lower part of the human body, Report for the Grant-in-Aid for Scientific Research by Ministry of Education, Science, Sports and Culture of Japan. Report no. 19300242, 25 Jun 2010.

13. M. Sato, S. Nishioka and T. Tamura, The Japan Society of Home Economics Annual meeting (ed 61th), Hyogo, Japan, 31 August 2009, paper no. 3 P-39. Tokyo: The Japan Society of Home Economics.

14. Y. Wang, Y. Cui, P. Zhang, et al., Text. Res. J., 81, 1113 (2011). 
15. R. Nayak, Woodh. Publ. Text., 241 (2017).

16. H. Kanai, K. Ogawa, T. Sasagawa, et al., Text. Res. J. (2021).

17. A. John, Anthropometric method -designing to fit the human body-: Human Factors and Ergonomics Society, Santa Monica, California, USA, 25 (1993).

18. H. Kanai, H. Tsuji, M. Kamijo, et al., J. Fiber Sci. Tech., 63, 159 (2007).

19. T. Nishimatsu, K. Tajima and H. Kanai, J. Text. Eng., 62, 7 (2016).

20. H\&K Ltd. Technical information, https://handkheisengp.co.jp/products/pdf/hei-cast_2870.pdf (2015, accessed 26 February 2015).

21. W. Maurel, D. Thalmann, Y. Wu, et al. Biomechanical Models for Soft Tissue Simulation. Springer Link, 121 (1998).

22. Nippon Thompson Co., Ltd. IKO technical service site, https://ikowb01.ikont.co.jp/technicalservice/ ikoc 0130.php?from=html (2020, accessed 14 June 2021).
23. Ito N. Compression feeling (translation from Japanese), Jpn. Res. Assn. Text. End-Uses, 36, 38 (1995).

24. Y. Doi and K. Kobayashi, Bull Health \& Sports Sci, Univ. Tsukuba, 11, 265 (1988).

25. S. Kawabata, The standardization and Analysis of Hand Evaluation $2^{\text {nd }}$ ed. Textile Machinery Society of Japan, Osaka (1980).

26. ISO 139:2005. Textile - Standard atmospheres for conditioning and testing.

27. T. Tamura, T. Koshiba and K. Hirata, Science on clothing environment (translation from Japanese), Tokyo: Kenpakusha (2004).

28. Research Committee of Sensory Evaluation. Sensory evaluation handbook. Tokyo: Union of Japanese Scientists and Engineers, 366 (1973). 